\title{
To block or not to block?
}

Contemporary joint replacement surgery has shown dramatic technical development in recent years. It is one of the most commonly performed procedures and is predicted to increase exponentially over the next decade. This has led to the development of enhanced recovery after surgery (ERAS) consisting of multimodal, multidisciplinary perioperative care pathways designed to reduce hospital length of stay through reduced morbidity and early ambulation.

An essential prerequisite for the success of an accelerated care pathway is early ambulation facilitated by the provision of dynamic pain relief (i.e. pain relief during movement). To achieve a shorter hospital length of stay, it is necessary that the pathway includes optimization and standardization of the preoperative, intraoperative, and postoperative pain management components.

An optimal multimodal pain therapy should be initiated in the preoperative period by the identification of patients who are at risk of greater pain intensity. Patient counselling and expectation management has been shown to improve pain relief and patient satisfaction [1]. Patients with high severity of preoperative pain are more likely to report chronic pain after both total hip and knee replacement and this association is five times stronger in patients undergoing total knee arthroplasty (TKA) compared to total hip arthroplasty (THA) [2]. Preoperative pain-on-movement is the strongest predictor of chronic pain-on-movement at 12 months in patients undergoing TKA. Preoperative pain-at-rest is weakly predictive of chronic pain-at-rest at 12 months in patients undergoing THA. Interestingly, acute postoperative pain-on-movement has not been associated with chronic pain after TKR or THR after adjusting for preoperative pain; however, acute painat-rest was associated with chronic pain after THR but not TKR after adjusting for preoperative pain [2].

Intra- and postoperative analgesia: the requirement for procedure specificity is now well recognized and universally accepted.

Address for correspondence:

Dr Gabriella Iohom

Department of Anaesthesia

and Intensive Care Medicine

Cork University Hospital

Wilton Road, Cork, Ireland

E-mail: gabriella.iohom@hse.ie
Local and/or regional analgesia techniques are cornerstones of a multimodal analgesia regimen because they provide excellent dynamic pain relief and reduce opioid consumption with the associated side effects. The duration of the sensory block may be extended by using continuous perineural catheters. However, a dense motor block is undesirable because it may hinder early mobilization and physical therapy. On the other hand, dynamic analgesia is required for the conduct of postoperative physical therapy.

The original article by McClain et al. in the current issue, while unique in evaluating physical therapists' preferences and opinions at a single institution, brings up several important issues with regards to the management of pain following joint arthroplasty [3]. Although the authors elude to the change in practice over time with regards to the perioperative analgesia plan, unfortunately no detailed drug information is provided for the spinal, peripheral block, periarticular infiltration or multimodal analgesia. Additional factors, such as the severity of postoperative pain, contribute to tailoring of the postoperative multimodal analgesia regimen to the actual surgical procedure. Postoperative pain after THA appeared less severe compared to that after TKA at all time points, with the highest median rest pain score preoperatively when an opioid-free spinal anaesthesia with minor analgesics and a postoperative morphine-based patient controlled analgesia (PCA) technique was used [4]. Both TKA and THA patients experienced their peak median in-patient pain score on the first postoperative day, when $47 \%$ of THA and $58 \%$ of TKA patients reported moderate-severe pain, which decreased to $11 \%$ and $43 \%$ respectively by postoperative day 3 [4]. This highlights the continuing need for better management of acute postoperative pain after arthroplasty, particularly on the first postoperative day, and particularly after TKR. Acute pain after orthopaedic surgery is even more severe on mobilisation, and interferes with walking ability, which is critical for postoperative recovery and the achievement of physiotherapy goals [5].

There is a large variability of analgesic techniques employed following joint arthroplasty. In an effort to standardise evidence-based procedure-specific analgesia, for THA, the PROSPECT group recommends 
the continuation of some form of regional analgesia (epidural only in patients at high cardiopulmonary risk, posterior lumbar plexus block or femoral block) following general anaesthesia only (and not after spinal anaesthesia), with postoperative multimodal analgesia encompassing systemic opioids, paracetamol and nonsteroidal analgesic drugs administered as appropriate for the level of postoperative pain [6].

For TKA, a femoral block is advocated by the PROSPECT group in conjunction with either general or opioid-free spinal anaesthesia. Specifically, insufficient procedure-specific evidence exists for combinations such as femoral and obturator or femoral and sciatic nerve blocks. Similarly, the lumbar plexus block is not recommended as a femoral nerve block is equally effective and is associated with fewer complications [6].

In recent years, more peripheral nerve blocks (saphenous, adductor canal block) have been shown to be as effective as femoral blocks following TKA without the associated motor weakness. Alternatively, weak $(0.03$ to $0.06 \%)$ and low volume $(3-5 \mathrm{ml} / \mathrm{h})$ local anaesthetic solutions have been shown to have no impact on lower limb muscle strength and to encourage patient participation in physical therapy on the first day post TKA due to improved pain and less opioid-induced side effects [7].

Local infiltration analgesia (LIA) is a systematic, extensive, meticulous, high-volume low-concentration periarticular infiltration technique (periosteum, capsule, ligaments, soft tissue) performed by the surgeon, born out by the necessity to provide prolonged analgesia with no motor block [8]. It has been shown to be an effective analgesic method following THA, similar or superior to no infiltration, placebo saline infiltration, intrathecal morphine and epidural analgesia $[9,10]$. In TKA, most trials reported reduced pain and reduced opioid requirements with LIA compared with a control group treated with placebo/no injection. Compared with femoral nerve block, epidural or intrathecal morphine, LIA provided similar or improved analgesia in the early postoperative period. LIA provides effective analgesia in the initial postoperative period after TKA in most randomized clinical trials even when combined with multimodal systemic analgesia. In contrast, LIA may have limited additional analgesic efficacy in THA when combined with a multimodal analgesic regimen [10].

The results of the current survey [3] are in line with the widespread practice of LIA as part of multimodal analgesia for TKA comprising minor analgesics, adjuvants (both pharmacological and non-pharmacological) and slow-release opioids administered around the clock, with immediate-release opioids for breakthrough pain. If thoroughly performed, there is no real advantage of associating a peripheral nerve block.
However, if there is any doubt over the efficacy of the operator-dependent LIA, the association of a saphenous, adductor canal or a femoral block with a dilute local anaesthetic solution may be justified.

For shoulder surgery, although the anterior deltopectoral incision lends itself to an interscalene anaesthestic or analgesic block with no implications for ambulation, alternative more peripheral analgesic blocks, such as supraclavicular, or suprascapular plus axillary blocks have been proposed. The latter are usually associated with a general anaesthetic for patient comfort. A more dilute local anaesthetic solution may limit the motor block while providing sufficient analgesia. Therefore, I believe that currently most centers associate a peripheral nerve block to their multimodal analgesia plan as a standard of care for total shoulder arthroplasty. It is unclear why only about half the physical therapists would prefer a nerve block, although this proportion was much higher compared to other joint replacements [3]. In line with the trend towards more peripheral blocks in other joint replacements, LIA is an emerging technique for shoulder arthroplasty [11].

Almost certainly, the future holds the discovery of additional factors which may influence postoperative pain. For example, little is known about rebound pain after effects of the local anaesthetic have dissipated. Currently, we strive to establish the best multimodal analgesia regimen for specific joint arthroplasties. Applying patient-centred paradigm shifts have the potential to lead to personalised analgesia regimens in the not too distant future. In the meantime, the most peripheral nerve block for a given procedure (beginning with periarticular infiltration where possible) and a dilute solution of local anaesthetic in the context of multimodal analgesia is but one piece of the jigsaw in the enhanced recovery after joint replacement.

\section{Conflict of interest}

Nothing to declare

Gabriella Iohom, PhD

Consultant Anaesthetist/Senior Lecturer Cork University Hospital/University College Cork Cork, Ireland

\section{Reference}

1. Lemay CA, Lewis CG, Singh JA, Franklin PD. Receipt of pain management information preoperatively is associated with improved functional gain after elective total joint arthroplasty. J Arthroplasty 2017; 32: 1763-1768. doi: 10.1016/j.arth. 2017.01 .028

2. Sayers A, Wylde V, Lenguerrand E, Beswick AD, GoobermanHill R, Pyke M, et al. Rest pain and movement-evoked pain as 
unique constructs in hip and knee replacements. Arthritis Care Res 2016; 68: 237-245. doi: 10.1002/acr.22656

3. McClain RL, Porter SB, Arnold SM, Robards CB. Peripheral nerve blocks and postoperative physical therapy: a single-institution survey of physical therapists' preferences and opinions. Rom J Anaesth Intensive Care 2017; 24: 115-124

4. Wylde V, Rooker J, Halliday L, Blom A. Acute postoperative pain at rest after arthroplasty: severity, sensory qualities and impact on sleep. Orthop Traumatol Surg Res 2011; 97: 139144. doi: 10.1016/j.otsr.2010.12.003

5. Dihle A, Helseth S, Kongsgaard UE, Paul SM, Miaskowski C. Using the American Pain Society's patient outcome questionnaire to evaluate the quality of postoperative pain management in a sample of Norwegian patients. J Pain 2006; 7 : 272-280. doi: 10.1016/j.jpain.2005.11.005

6. www.postoppain.org (accessed on September $4^{\text {th }}$ 2017)

7. Liu Q, Chelly JE, Williams J, Gold MS. Impact of peripheral nerve block with low dose local anesthetics on analgesia and functional outcomes following total knee arthroplasty: a retrospective study. Pain Med 2015; 16: 998-1006. doi: $10.1111 /$ pme. 12652
8. Joshi GP, Cushner FD, Barrington JW, Lombardi AV Jr, Long WJ, Springer BD, et al. Techniques for periarticular infiltration with liposomal bupivacaine for the management of pain after hip and knee arthroplasty: a consensus recommendation. J Surg Orthop Adv 2015; 24: 27-35

9. McCarthy D, Iohom G. Local Infiltration Analgesia for Postoperative Pain Control following Total Hip Arthroplasty: A Systematic Review. Anesthesiol Res Pract 2012; 2012: 709531. doi: $10.1155 / 2012 / 709531$

10. Andersen LØ, Kehlet H. Analgesic efficacy of local infiltration analgesia in hip and knee arthroplasty: a systematic review. $\mathrm{Br}$ J Anaesth 2014; 113: 360-374. doi: 10.1093/bja/aeu 155

11. Sabesan VJ, Shahriar R, Petersen-Fitts GR, Whaley JD, BouAkl T, Sweet M, et al. A prospective randomized controlled trial to identify the optimal postoperative pain management in shoulder arthroplasty: liposomal bupivacaine versus continuous interscalene catheter. J Shoulder Elbow Surg 2017. pii: S10582746(17)30393-2. doi: 10.1016/j.jse.2017.06.044. [Epub ahead of print]

Rom J Anaesth Intensive Care 2017; 24: 83-85 